Ambient Science, 2018: Vol. 05(Sp1); 51-55

DOI:10.21276/ambi.2018.05.sp1.ra01

\title{
Risk Factors for Wound Infectious Complications in Orthopedic Surgery Ward: A Case-control Study of Iran
}

\section{Marzieh Kazeminejad', Mohsen Hojat', Sareh Abdollahifard ${ }^{2 *}$, Mahdi Karimiar ${ }^{2}$, Farzaneh Alipour ${ }^{3}$, Shohreh Javadpoor ${ }^{3}$, Majid Maddahfar ${ }^{4}$}

${ }^{1}$ Research Center for Noncommunicable Diseases, Jahrom University of Medical Sciences, Jahrom, Iran

${ }^{2}$ Research Center for Social Determinants of Health, Jahrom University of Medical Sciences, Jahrom, Iran

3 Jahrom University of Medical Sciences, Jahrom, Iran

${ }^{4}$ BHOWCO Trading GmbH, Frankfurt am Main, Germany

Study Area:Jahrom, Iran

Coordinates: $28^{\circ} 30^{\prime} \mathrm{N} ; 53^{\circ} 33^{\prime} \mathrm{E}$

Key words: Apparent diffusion coefficient map, MR imaging.

A case-control study was approved by ethics committee of Jahrom University of medical sciences, Jahrom, Iran. Code: (ethics.HSR.9o / 6).

\section{Introduction:}

Surgical wound infections are a common cause of nosocomial infection. The United States Centers for Disease Control and Prevention (CDC) has developed criteria that define Surgical Site Infection (SSI) as infection related to an operative procedure that occurs at or near the surgical incision within 30 days of the procedure, or within 90 days if prosthetic material is implanted at surgery (Horan et al., 1992). SSIs are often localized to the incision site but can also extend into deeper adjacent structures. However, the overall risk of SSI is low; it is estimated that SSIs develop in 2 to 5 percent while monitored in 30 million patients undergoing surgical procedures each year (Lewis et al., 2013; Owens et al., 2014).

The SSI rates in ambulatory surgical settings are relatively low; one study noted overall rates for 14 and 30 days were 3.1 and 4.8 per 1000 procedures (Boyce et al., 1990) where antimicrobial prophylaxis and adjunctive measures for prevention of surgical site infection were discussed separately. Surgical site infections (SSIs) are associated with substantial morbidity/mortality, prolonged hospital stay, and increased cost (Poulsen et al., 1994; Vegas et al., 1993; Whitehouse et al., 2002). Among

\section{Abstract}

Wound infections are among the most common reasons why people seek medical advice. In a case-control study, we compared 37 patients with postoperative wound infection in orthopedic-surgical site with 37 persons without infection. During the study we reviewed medical records of the patients who had orthopedic surgery, and collected data regarding the complete treatment and follow up process after discharge, mainly focusing on possible risk factors in the onset of SSIs (surgical site infection). We found the risk of SSI, pre and posterior surgical approach were independently associated with a higher risk of infections. The significant differences were seen between hospitalization period and SSI, hematoma at the site of surgery and types of surgeries done besides orthopedics. The finding showed that prevalence of SSI was $8.8 \%$ and it is influenced by the demographic characteristic of patients, injury characteristics, compliance with care protocols before, during and after surgery, factors related to hospitals and staff and types of wound and surgeries.

patients with SSI who died in the post-operative periods, deaths were seen to be directly related to SSI in more than $75 \%$ of cases. In one paired case-control study of SSI following orthopedic procedures, the occurrence of SSI accounted for a median increase in a hospital stay of 14 days, an increase in the rate of re-hospitalization was just double, and increased total costs of more than 300\% (Perencevich et al., 2003).

Wound infection which is def ined as the infection of a wound caused by physical injury of the skin as a result of penetrating trauma from plants, animals, guns, knives or other objects is increasingly becoming a global concern with an increasing awareness of its cost burden (Horan et al., 1992; Lewis et al. 2013). Wound infections are among the most common reasons why people seek medical advice and in more serious acute infections, may require hospitalization. These infections account for around $7 \%$ $10 \%$ of hospitalized patients in the United States (Owens et al., 2014). Wound infection related to surgical wounds are considered to be one of the most common nosocomial infections and are generally a high cause of morbidity and mortality (Lewis et al. 2013; Owens et al., 2014; Boyce et al., 1990; Poulsen et al., 1994 \&Vegas et al., 1993).

A wide range of factors have been evidenced to 
influence wound infection. Some of such factors include pre-existing illness, wound class, wound contamination, age factor, malignancy, metabolic diseases, malnutrition, immunosuppression, cigarette smoking, remote site infection, length of surgical operation, emergency procedures and long duration of pre and postoperative hospitalization amongst others (Poulsen et al., 1994; Vegas et al., 1993; Whitehouse et al., 2002 \& Perencevich et al., 2003). To reduce these complications, it is important to establish the risk factors that increase the chances of the incidence using multivariate analysis. Though the issue has already been addressed for orthopedic wound surgery (Owens et al., 2014), but it has not been well studied for orthopedic wound surgery. However, the efficiency of antibiotic prophylaxis for reducing postoperative infectious complications has been demonstrated in the previous prospective, randomized studies (Vegas et al., 1993; Whitehouse et al., 2002 \& Perencevich et al., 2003), controversy still exists about which specific antibiotic agent to use (Lewis et al., 2013). As per the current status, no studies yet consider postoperative global infectious complications including orthopedic infectious complications. The risk factors for SSI and for global infectious complications may differ. Our study estimated the risk factors for SSI and for global infectious complications in orthopedic surgery ward (in patients who received antibiotic prophylaxis). Identification of risk factors in the perioperative period may allow for a reduction in the rate of postoperative infectious complications.

\section{Methodology:}

This study was conducted in orthopedic surgery ward of a hospital affiliated to Jahrom University of Medical Sciences during 2010 March to 2011 March.

For the study, 50 patients were considered in each group. Inclusion criteria were Iranian nationality, having normal BMI, going through orthopedics surgery and not having other surgeries in past 6 months before initiation of our study. Exclusion criteria were not having compliance of the patients, not having access to patient medical record and death after surgery, need to intensive care pre or post operation.

Post-operative infection is defined as an infection occurring within 1 month after operation and infection of wound accompanying discharge (abnormal volume, color, and odor) and positive culture of the wound. At first, sampling method was done following the purpose of the study. The researcher filled out the questionnaire related to demographic variables in orthopedics surgery ward and then inclusion and exclusion criteria were given to nurses of the mentioned wards and the questionnaires were finally completed and collected. The face and content validity of the tool was obtained with the help of 10 faculty members at the university (IS: .89, CVI: .93, CVR: .85) and the reliability was calculated using the test-retest method $(\alpha=.83)$.

Both case and control groups were matched concerning age, gender, season of their admission and referred to the hospital, type of fracture, site of fracture and type of antibiotics administered. Total 13 patients were excluded finally, 3 patients due to incomplete medical documents, 3 of them were dead and 7 were not interested to participate in the study. Finally, assessment of possible causes of postoperative infection was done on 2 groups (case group: 37 Orthopedic patients suffered from SSI and control group: 37 Orthopedic patients without SSI). These 3 categories of cause of infection were mentioned as below:

i) Cause related to patients' personal data (age, gender, occupation, accommodation place and previous infection).

ii) Cause related to the trauma (type and site of fracture, hematoma, time of trauma, type of accident and trauma, size of fracture and wound)

iii) Cause related to the hospital's management issues (preparation method before operating, ward in which patients admitted to it, type of palatine, time taken for transferring patient to the surgery ward, duration of hospitalization, frequency of changing dressing and antibiotic administration method).

Required documents were completed by interviewing with patients and some of the required remaining data were taken from the medical records of the patients. Data were analyzed through SPSS v 16 and ' $k$ ' square test (risk factors in groups), Mann- Whitney 'u' test, and 't' student (differences of the 2 group) were used.

\section{Results:}

In this study, a comparison between a group of 37 patients from orthopedic surgery ward and postoperative wound infection and 37 patients which were considered as the control group were selected from those who admitted in orthopedics ward having no postoperative wound infection. Obtained results are as follows:

a) Risk factors related to causes of trauma: results of the study showed significant difference in the case and control groups regarding hematoma( $\mathrm{p}<0.05)$. Total $81.1 \%$ of the case group and $97.3 \%$ of the control group had the hematoma. No statistically significant difference was found between 2 groups regarding bleeding amount and injury occurrence severity. About $78.4 \%$ of the case group and $76.5 \%$ of the control group reported bleeding. It was demonstrated that most of the injuries occurred in both groups were considered as moderate and severe. Total13.5\% of the injuries of the case group were considered as necessary and $\mathbf{2 . 7 \%}$ were life-threatening. Hematoma in wound decrease $88 \%$ Bleeding chance in patients (OR o.119) (tableı).

b) Risk factors related to the patients: results of the 
RESEARCH ARTICLE

study showed statistically no significant difference between both the groups concerning addiction, fracture and blood product transfusion ( $\mathrm{p}>0.05$ ).

Total $75.5 \%$ of the case group and $86.5 \%$ of the control group used opium, whereas $89.2 \%$ of the case group and $91.2 \%$ of the control group were addicted. Further, $97.3 \%$ of the case group and $94.6 \%$ of the control group had a fracture. About $64.9 \%$ of the case group and $83.8 \%$ of the control group had blood product transfusion. (Table 2).

Complete $100 \%$ of the patients in both groups had a previous postoperative infection, urinary and fecal incontinence, urinary tract infection, respiratory tract infection and septicemia. In the case group, $56.8 \%$ of the patients used hochi and $43.2 \%$ used packed cell. In the control group, $83.8 \%$ of the patients used hochi and $16.2 \%$ used packed cell. Fractures increased the risk of bleeding double (OR 2.057).

Table-1: Significant Level of Risk Factor in Wound Infection

\begin{tabular}{|c|c|c|c|c|c|}
\hline \multirow{3}{*}{ Risk Factors } & \multicolumn{4}{|c|}{ Group } & \multirow{3}{*}{$\begin{array}{l}\text { OR } \\
\text { (CI 95\%) }\end{array}$} \\
\hline & \multicolumn{2}{|c|}{ Infected } & \multicolumn{2}{|c|}{ Non-Infected } & \\
\hline & $\mathrm{N}$ & $\%$ & $\mathrm{~N}$ & $\%$ & \\
\hline \multicolumn{6}{|l|}{ Hematoma } \\
\hline Positive & 30 & 81.1 & 36 & $97 \cdot 3$ & $0.119(0.014-<0.025$ \\
\hline Negative & 7 & 18.9 & 1 & 2.7 & 1.23) \\
\hline \multicolumn{6}{|l|}{ Bleeding } \\
\hline Positive & 29 & 78.4 & 32 & 86.5 & $0.566(0.166<0.359$ \\
\hline Negative & 8 & 21.6 & 5 & 13.5 & 1.92) \\
\hline \multicolumn{6}{|c|}{ The damage to the Body } \\
\hline Mild & 15 & 40.5 & 15 & 40.5 & $0.744(0.482<0.112$ \\
\hline Moderate & 12 & 32.4 & 14 & 37.8 & -1.147 \\
\hline Sever & 4 & 10.8 & 8 & 21.6 & \\
\hline Serious & 1 & 2.7 & o & 0.0 & \\
\hline Critical & 5 & 13.5 & o & 0.0 & \\
\hline Deadly & o & 0.0 & o & 0.0 & \\
\hline
\end{tabular}

\begin{tabular}{|c|c|c|c|c|c|}
\hline \multirow[t]{3}{*}{ Risk Factors } & \multicolumn{4}{|c|}{ Group } & \multirow{3}{*}{$\begin{array}{l}\text { OR } \\
(\mathrm{CI} 95 \%)\end{array}$} \\
\hline & \multicolumn{2}{|c|}{ Infected } & \multicolumn{2}{|c|}{ Non-Infected } & \\
\hline & $\mathrm{N}$ & $\%$ & $\mathrm{~N}$ & $\%$ & \\
\hline \multicolumn{6}{|l|}{ Smoking } \\
\hline Positive & 28 & $75 \cdot 7$ & 32 & 86.5 & $0.486(0.146<0.235$ \\
\hline Negative & 9 & $24 \cdot 3$ & 5 & 13.5 & $-1.62)$ \\
\hline \multicolumn{6}{|l|}{ Addiction } \\
\hline Positive & 33 & 89.2 & 34 & 91.9 & $0.728(0.151<0.691$ \\
\hline Negative & 4 & 10.8 & 3 & 8.1 & $-3.505)$ \\
\hline \multicolumn{6}{|l|}{ Fractures } \\
\hline Positive & 36 & $97 \cdot 3$ & 35 & 94.6 & \multirow{2}{*}{$\begin{array}{l}2.057(0.178<0.556 \\
-23.72)\end{array}$} \\
\hline Negative & 1 & 2.7 & 2 & $5 \cdot 4$ & \\
\hline \multicolumn{6}{|c|}{ Blood Transfusions } \\
\hline Positive & 24 & 64.9 & 31 & 83.8 & \multirow{2}{*}{$\begin{array}{l}0.357(0.118-<0.062 \\
1.078)\end{array}$} \\
\hline Negative & 13 & 35.1 & 6 & 16.2 & \\
\hline
\end{tabular}

In the case group, WBC count before the operation was $10.41 \pm 2.75$ and $9.75 \pm 3.45$ was after the operation. In the control group, WBC count was $8.51 \pm 3.08$ before the operation and $8.19 \pm 2.63$ after the operation. It revealed a
Ambient Science, 2018: Vol. 05(Sp1); 51-55 DOI:10.21276/ambi.2018.05.sp1.ra01

statistically significant difference in WBC count before and after operation between the 2 groups (table- 3 ).

Table-3: Risk Factors of Surgical Site Infection

\begin{tabular}{lccccc}
\hline Group & $\mathrm{N}$ & Mean & Std. Dev. & $\mathrm{t}$ & $\mathrm{p}$-value \\
\hline The lastWBC Test of Injured before surgery & & \\
Infected & 28 & 10.3321 & 2.73781 & 2.379 & $\mathbf{0 . 0 2 1}$ \\
Non-Infected & 30 & 8.5067 & 3.07974 & & \\
The last WBC Test of Injured after surgery & & \\
Infected & 28 & 9.7893 & 3.45429 & 2.012 & 0.049 \\
Non-Infected & 31 & 8.1903 & 2.63000 & & \\
\hline
\end{tabular}

c) Risk factor related to the hospital: the results reveal that, in the case group accompanying person $86.5 \%$ and personnel $13.5 \%$ whereas in the control group, accompanying person $83.8 \%$ and personnel $13.5 \%$ and the patient $\mathbf{2 . 7 \%}$ were having risk factors of infection. The education level of the shaver, in the case group, was $51.4 \%$ below diploma and $29.6 \%$ diploma. Total $92.2 \%$ of the case group were admitted to the emergency, surgery and ICU wards (table 4$)$.

Table-4: Risk Factors of Orthopedics Wound Infection

\begin{tabular}{lllll}
\hline \multicolumn{5}{c}{ Group } \\
Parameters & \multicolumn{2}{c}{ Infected } & \multicolumn{2}{c}{ Non-Infected } \\
& $\mathrm{N}$ & $\%$ & $\mathrm{~N}$ & $\%$ \\
\hline One who is shaved & & & & \\
Patient & $\mathrm{O}$ & $0.0 \%$ & 1 & $2.7 \%$ \\
Even with patient & 32 & $86.5 \%$ & 31 & $83.8 \%$ \\
Hospital Staff & 5 & $13.5 \%$ & 5 & $13.5 \%$ \\
Education of One who is shaved & & & \\
Uneducated & 5 & $13.5 \%$ & 4 & $11.1 \%$ \\
Below-diploma & 19 & $51.4 \%$ & 17 & $47.2 \%$ \\
Diploma & 11 & $29.7 \%$ & 12 & $33.3 \%$ \\
Bachelor & 0 & $0.0 \%$ & 2 & $5.6 \%$ \\
Master & 2 & $5.4 \%$ & 1 & $2.8 \%$ \\
Shift the way & & & & \\
Morning & 26 & $70.3 \%$ & 24 & $64.9 \%$ \\
Evening & 8 & $21.6 \%$ & 11 & $29.7 \%$ \\
Night & 3 & $8.1 \%$ & 2 & $5.4 \%$ \\
Wards before surgery & & & & \\
Emergency & 12 & $32.4 \%$ & 21 & $56.8 \%$ \\
Surgery & 16 & $43.2 \%$ & 4 & $10.8 \%$ \\
ICU & 7 & $18.9 \%$ & 8 & $21.6 \%$ \\
CCU & 0 & $0.0 \%$ & 3 & $8.1 \%$ \\
Internal ward & 2 & $5.4 \%$ & 1 & $2.7 \%$ \\
\hline
\end{tabular}

There is a statistically signif icant difference between 2 groups concerning the duration of time before the operation, but no signif icant difference between 2 groups concerning the duration of time after the operation has been revealed. However, there is a statistically significant difference exist between 2 groups concerning dosage of antibiotic before the operation. Similarly, there is also a statistically signif icant difference reveal between 2 groups concerning dosage of antibiotic after operation (table 5 ). 
Table-5: Signif icant Level of Surgical Site Infection Risk Factors

\begin{tabular}{|c|c|c|c|c|}
\hline Group & $\mathrm{N}$ & Mean+SD & Mann-Whitney & $\mathrm{p}$ \\
\hline \multicolumn{5}{|c|}{ Duration of hospitalization before surgery } \\
\hline Infected & 37 & $2 \pm 1$ & \multirow[t]{2}{*}{$415 \cdot 50$} & \multirow[t]{2}{*}{$>0.001$} \\
\hline Non-Infected & 37 & $1 \pm 0$ & & \\
\hline \multicolumn{5}{|c|}{ Duration of hospitalization after surgery } \\
\hline Infected & 37 & $8 \pm 7$ & \multirow[t]{2}{*}{$567 \cdot 5$} & \multirow[t]{2}{*}{$>0.200$} \\
\hline Non-Infected & 37 & $5 \pm 3$ & & \\
\hline \multicolumn{5}{|c|}{ Dosage of Antibiotics before surgery } \\
\hline Infected & 37 & $6 \pm 7$ & \multirow[t]{2}{*}{589.0} & \multirow[t]{2}{*}{$>0.286$} \\
\hline Non-Infected & 37 & $5 \pm 6$ & & \\
\hline \multicolumn{5}{|c|}{ Dosage of Antibiotics after surgery } \\
\hline Infected & 37 & $23 \pm 22$ & \multirow[t]{2}{*}{488.0} & \multirow[t]{2}{*}{$>0.032$} \\
\hline Non-Infected & 37 & $15 \pm 12$ & & \\
\hline
\end{tabular}

Invasive action in the case group was mentioned as $37.5 \%$ suction and $62.5 \%$ intubation. There was a statistically significant difference between 2 groups regarding duration of hospitalization, type of operation, duration of surgery (table 6).

Table-6: Risk Factors of Wound Infections

\begin{tabular}{|c|c|c|c|c|}
\hline Group & $\mathrm{N}$ & Mean \pm SD & Mann-Whitney & $\mathrm{p}$ \\
\hline \multicolumn{5}{|c|}{ A few hours before the procedure is performed Shaved } \\
\hline Infected & 37 & $7 \pm 11$ & \multirow[t]{3}{*}{$593 \cdot 50$} & \multirow[t]{2}{*}{$<0.321$} \\
\hline Non-Infected & 37 & $13 \pm 19$ & & \\
\hline \multicolumn{4}{|c|}{ Case after how many days/hours after admitted to hospital and. } & \\
\hline Infected & 37 & $3 \pm 2$ & \multirow[t]{2}{*}{$555 \cdot 5$} & \multirow{2}{*}{$<0.153$} \\
\hline Non-Infected & & $3 \pm 2$ & & \\
\hline \multicolumn{5}{|c|}{ The patient was discharged a few days after surgery and ..... } \\
\hline Infected & 37 & $8 \pm 8$ & \multirow[t]{2}{*}{478.5} & \multirow[t]{2}{*}{$<0.021$} \\
\hline Non-Infected & 37 & $4 \pm 3$ & & \\
\hline \multicolumn{5}{|c|}{ In total, the patient was hospitalized for several days and .... } \\
\hline Infected & 37 & $11 \pm 8$ & \multirow[t]{2}{*}{552.0} & \multirow[t]{2}{*}{$<0.150$} \\
\hline Non-Infected & 37 & $7 \pm 3$ & & \\
\hline \multicolumn{5}{|c|}{ How much duration in hours the surgery took and ..... } \\
\hline Infected & 37 & $100 \pm 50$ & \multirow[t]{2}{*}{$439 \cdot 5$} & \multirow[t]{2}{*}{$<0.006$} \\
\hline Non-Infected & 37 & $71 \pm 25$ & & \\
\hline \multicolumn{5}{|c|}{$\begin{array}{l}\text { The number of operations carried out in the same shift in the } \\
\text { operating room and ..... }\end{array}$} \\
\hline Infected & 37 & $11 \pm 6$ & \multirow[t]{2}{*}{626.0} & \multirow[t]{2}{*}{$<0.523$} \\
\hline Non-Infected & 37 & $12 \pm 22$ & & \\
\hline \multicolumn{5}{|c|}{$\begin{array}{l}\text { The type of operation carried out in the same shift in the } \\
\text { operating room, except for orthopedic surgery and.... }\end{array}$} \\
\hline Infected & 37 & $4 \pm 3$ & \multirow[t]{2}{*}{372.0} & \multirow[t]{2}{*}{$<0.001$} \\
\hline Non-Infected & 37 & $2 \pm 2$ & & \\
\hline \multicolumn{5}{|c|}{ The patient had several operations on that day and.... } \\
\hline Infected & 37 & $3 \pm 2$ & \multirow[t]{2}{*}{389.5} & \multirow[t]{2}{*}{$<0.001$} \\
\hline Non-Infected & 37 & $5 \pm 3$ & & \\
\hline \multicolumn{5}{|c|}{ The average operating room personnel shifts in the same month } \\
\hline Infected & 37 & $211 \pm 2$ & \multirow[t]{2}{*}{684.5} & \multirow[t]{2}{*}{$<1.0$} \\
\hline Non-Infected & 37 & $211 \pm 2$ & & \\
\hline
\end{tabular}

There is statistically no significant difference regarding the frequency of changing dressing before the operation has been evidenced. On the other hand, a statistically significant difference was shown in the frequency of changing dressing after the operation. In the case group, total $91.9 \%$ and $59.5 \%$ in the control group, changing dressing at home was required (table 7 ).
Table-7: Nursing and Changing Dressing before operation

\begin{tabular}{|c|c|c|c|c|c|c|}
\hline \multirow[t]{3}{*}{ Risk Factors } & \multicolumn{4}{|c|}{ Group } & \multirow{3}{*}{\multicolumn{2}{|c|}{$\begin{array}{l}\text { OR } \\
(\mathrm{CI} 95 \%)\end{array}$}} \\
\hline & \multicolumn{2}{|c|}{ Infected } & \multicolumn{2}{|c|}{ Non-Infected } & & \\
\hline & $\mathrm{N}$ & $\%$ & $\mathrm{~N}$ & $\%$ & & \\
\hline \multicolumn{7}{|c|}{ Frequency of dressing change on the day before surgery } \\
\hline o & 21 & 56.8 & 19 & 51.4 & \multirow{4}{*}{$\begin{array}{l}1.0 \\
(0.014-1.23)\end{array}$} & $<0.708$ \\
\hline 1 & 14 & 37.8 & 17 & $45 \cdot 9$ & & \\
\hline 2 & 1 & 2.7 & 1 & 2.7 & & \\
\hline 3 & 1 & 2.7 & o & 0.0 & & \\
\hline \multicolumn{7}{|c|}{ Frequency of dressing change after surgery a day } \\
\hline o & o & 0.0 & 1 & 2.7 & - & $<0.008$ \\
\hline 1 & 29 & 78.4 & 36 & $97 \cdot 3$ & & \\
\hline 2 & 8 & 21.6 & o & 0.0 & & \\
\hline
\end{tabular}

\section{Discussion:}

Surgical site infections are one of the most common nosocomial infections besides other systemic infections (Al-Mulhim et al., 2014). According to the first national prevalence study of nosocomial infections, surgical site infections were the most prevalent infections and is one of the highest prevalence rates of nosocomial infections in the orthopedic wards (Al-Mulhim et al., 2014). Maksimovic, et al. (2008) in their study revealed that a high incidence of surgical site infections in orthopedic patients and established risk factors related to surgical site infection. Smoking didn't reveal any signif icant difference in the above-mentioned study which is in agreement with ourfinding.

Further, open reduction fracture and hours between shaving and operation were seen as another factor which influences SSI. These factors were also considered in our study but no statistically significant differences were seen. Numbers of staff in the operating room and presence of drains were the other parameters evaluated in the mentioned study that they affected SSI significantly (Maksimovic et al., 2008). In our study dosage of antibiotics before surgery did not reveal any statistically significant difference. Although Bratzler \& Houck (2005) in their study established that dosage and duration of antibiotics before the surgery is effective.

Ercole et al. (2011) evaluated the SSI infection with evaluated risk factors and showed no significant difference between preoperative hospitalization time and SSI but got a significant difference between the duration of operation and SSI. On the other hand, according to a study by Ercole et al.(2011) duration of surgery and SSI are directly connected. Our results strengthened the above findings. Elongation of surgery might decrease infection and might increase fatigue of staff and which perhaps increase SSI. Statistically significant difference between hospitali-zation period and SSI somehow indicating the same.

Further, some other parameters were given interesting results in our study. Hematoma at the site of surgery and types of surgeries done besides orthopedics 
simultaneously and waiting times spent were effective. Hojat et al. (2013) in their study determined the risk factors of SSI in orthopedics wards and the results of their study strengthened our findings and they revealed that duration of hospitalization and existence of hematoma signif icantlyaffect SSI.

The finding showed that prevalence of SSI was $8.8 \%$ and it is influenced by the demographic characteristic of patients, injury characteristics, compliance with care protocols before, during and after surgery, factors related to hospitals and staff and types of wound and surgeries. These controversies in the results might be due to variation in operation rooms in developed and developing countries and personal differences. Thus, further studies are also required to support our findings and to evaluate the risk factors more precisely. Although the incidence of postoperative infection depends on the incidence of uncontrollable factors such as patient demographics, risk factors, and injury characteristics but compliance with care protocols before, during and after surgery, helps to prevent the incidence of even worst possible cases of infection.

Some more studies are required to support our findings and to consider other parameters such as usage of drains and numbers of staff.

\section{Acknowledgment:}

The authors are thankful to the subjects (patients) who had participated in this study and allowed us to use their medical data as a part of this report.

\section{References:}

Al-Mulhim F.A., Baragbah, M.A., Sadat-Ali, M., Alomran, A.S., Azam, M.Q. (2014): Prevalence of Surgical Site Infection in Orthopedic Surgery: A 5-year Analysis. Int. Surg., 99(3): 264268.

Boyce, J.M., Potter-Bynoe, G. \& Dziobek, L. (1990): Hospital reimbursement patterns among patients with surgical wound infections following open heart surgery. Infect. Control Hosp. Epidemiol., 11(2):89-93.

Bratzler, D.W. \& Houck, P.M. (2005): Antimicrobial Prophylaxis for Surgery: An Advisory Statement from the National Surgical Infection Prevention Project, Am. I. Surg.. 189(4):395-404.
Ercole, F.F., Franco, L.M.C., Macieira, T.G.R., Wenceslau, L.C.C., de Resende, H.I.N. \& Chianca, T.C.M. (2011): Risk of surgical site infection in patients undergoing orthopedic surgery. Revista Latino-Americana de Enfermagem.19(6):1362-1368.

Hojat, M., Karimyar-Jahromi, M., Salehifard, A., Keshaei, N. (2013): Assessment the Effect of Various Factors on Theincidence of Surgical Site Infection in Orthopedic patients. Iran. J. Surg., 21(2):53-60.

Horan, T.C., Gaynes, R.P., Martone, W.J., Jarvis, W.R. \& Emori, T.G.(1992): Definitions of nosocomial surgical site infections. Infect. Control Hosp. Epidemiol. 13(10):606-6o8.

Lewis, S.S., Moehring, R.W., Chen, L.F., Sexton, D.J. \& Anderson, D. (2013): Assessing the relative burden of hospital-acquired infections in a network of community hospitals. Infect. Control Hosp. Epidemiol., 34(11):1229-1230.

Maksimovic, J., Markoviæ-Deniæ, L., Bumbaširevic, M., Marinkovic, J. \& Vlajinac, H. (2008): Surgical Site Infections in Orthopedic Patients: Prospective Cohort Study. Croat. Med.J., 49(1):58-65.

Owens, P.L., Barrett, M.L., Raetzman, S., Maggard-Gibbons, M. \& Steiner, C.A. (2014): Surgical site infections following ambulatory surgery procedures. JAMA.311(7):709-716.

Perencevich, E.N., Sands, K.E., Cosgrove, S.E., Guadagnoli, E., Meara, E. \& Platt, R. (2003): Health and economic impact of surgical site infections diagnosed after hospital discharge. Emerg. Infect. Dis., 9(2):196-203.

Poulsen, K.B., Bremmelgaard, A., Sørensen, A.I., Raahave, D., Petersen, J.V. (1994): Estimated costs of postoperative wound infections. A case-control study of marginal hospital and social security costs. Epidemiol. Infect., 113(2):283-295

Vegas, A.A., Jodra, V.M. \& García, M.L. (1993): Nosocomial infection in surgery wards: a controlled study of increased duration of hospital stays and direct cost of hospitalization. Eur. J. Epidemiol., 9(5):504-510

Whitehouse, J.D., Friedman, N.D., Kirkland, K.B., Richardson, W.J. \& Sexton, D. (2002): The impact of surgical-site infections following orthopedic surgery at a community hospital and a university hospital: adverse quality of life, excess length of stay, and extra cost. L. Infect. Control Hosp. Epidemiol., 23(4):183-189. 